\title{
Research Article \\ Spectral Approximation of an Oldroyd Liquid Draining down a Porous Vertical Surface
}

\author{
F. Talay Akyildiz, ${ }^{1}$ Mehmet Emir Koksal, ${ }^{2}$ and Nurhan Kaplan ${ }^{3}$ \\ ${ }^{1}$ Department of Mathematics, University of Gaziantep, 27310 Gaziantep, Turkey \\ ${ }^{2}$ Department of Elementary Mathematics Education, Mevlana University, 42003 Konya, Turkey \\ ${ }^{3}$ Department of Mathematics, Nigde University, 51240 Nigde, Turkey \\ Correspondence should be addressed to F. Talay Akyildiz, fakyildiz@pi.ac.ae
}

Received 29 June 2011; Revised 5 October 2011; Accepted 10 October 2011

Academic Editor: Carlo Piccardi

Copyright (C) 2011 F. Talay Akyildiz et al. This is an open access article distributed under the Creative Commons Attribution License, which permits unrestricted use, distribution, and reproduction in any medium, provided the original work is properly cited.

Consideration is given to the free drainage of an Oldroyd four-constant liquid from a vertical porous surface. The governing systems of quasilinear partial differential equations are solved by the Fourier-Galerkin spectral method. It is shown that Fourier-Galerkin approximations are convergent with spectral accuracy. An efficient and accurate algorithm based on the Fourier-Galerkin approximations for the governing system of quasilinear partial differential equations is developed and implemented. Numerical results indicating the high accuracy and effectiveness of this algorithm are presented. The effect of the material parameters, elasticity, and porous medium constant on the centerline velocity and drainage rate is discussed.

\section{Introduction}

Thin-film drainage down porous vertical surfaces is important in industry. Draining films occur in processes as diverse as dip coating, electroplating, enameling, emptying storage vessels, and oil recovery mechanisms [1,2]. Spectral projection and corresponding error analysis of the system of nonlinear partial differential equations arising in the free drainage startup flow of Oldroyd four constant liquids over a porous vertical surface is considered.

Literature review reveals that this problem is not considered. But for the case of impermeable wall, Goshawk and Waters [3] and Pennington and Waters [4] investigated the drainage of an Oldroyd four-constant liquid from a vertical surface via a finite difference method. But the problem they consider is a special case of the expended investigation in this paper, and error analysis is not explored in their work. Again, for case of steady flow (or startup phase neglected), the literature more richer, in this case, Keeley et al. [5] investigate the drainage of thin films of non-Newtonian liquids from vertical surface, and the behavior of the Phan Thain-Tanner models are investigated in detail $[6,7]$. In the present study, Galerkin's 
method of the system of quasilinear partial differential equations governing the free drainage problem is investigated for a porous vertical surface. It is shown that method converges and that the convergence is not at all dependent on whether or not the physical parameters of the problem assume special values. The paper is organized as follows. The problem is defined in Section 2, and some basic results on Fourier approximations are given. A suitable FourierGalerkin approximation for the problem under consideration is proposed in Section 3 and error analysis given following [8-11]. Efficient and robust algorithms for the problem under consideration are constructed and numerical results presented in Section 4.

\section{Mathematical Formulation and Preliminary Results on Fourier Approximation}

Consider a thin liquid film draining down a flat porous vertical surface defined by Cartesian coordinates $(x, y, z)$. The $x$ axis points vertically downwards, the solid surface lies in the plane $y=0$ with the thickness of the liquid film measured in the positive $y$ direction, and the $z$ axis is positioned perpendicular to the gravitational force completing a set of righthanded axes. The nondimensionalized equations of motion and the dimensionless Oldroyd four constant constitutive model form a quasilinear system of PDEs, where (details can be found in $[3,4,12,13]$ for the interested reader)

$$
\begin{gathered}
\frac{\partial S_{x y}}{\partial y}=\frac{\partial u}{\partial t}-1+\alpha^{2} u \\
S_{x y}+S_{1} \frac{\partial S_{x y}}{\partial t}+\frac{1}{2} \mu_{1} S_{x x} \frac{\partial u}{\partial y}=\left(\frac{\partial u}{\partial y}+S_{2} \frac{\partial^{2} u}{\partial y \partial t}\right), \\
S_{x x}+S_{1} \frac{\partial S_{x x}}{\partial t}-2 S_{1} S_{x y} \frac{\partial u}{\partial y}=-2 S_{2}\left(\frac{\partial u}{\partial y}\right)^{2}
\end{gathered}
$$

where above we used the following dimensionless parameters as in [3]

$$
\begin{gathered}
y=Y\left(\frac{g}{v^{2}}\right)^{1 / 3}, \quad x=x\left(\frac{g}{v^{2}}\right)^{1 / 3}, \quad h=H(x, t) y=Y\left(\frac{g}{v^{2}}\right)^{1 / 3}, \quad t=T y=Y\left(\frac{g^{2}}{v}\right)^{1 / 3}, \\
S_{1}=\lambda_{1}\left(\frac{g^{2}}{v}\right)^{1 / 3}, \quad S_{2}=\lambda_{2}\left(\frac{g^{2}}{v}\right)^{1 / 3}, \quad u=\frac{U}{(v g)^{1 / 3}}, \quad v=\frac{V}{(v g)^{1 / 3}}, \\
S_{i j}=P_{i j} \frac{\mu g}{v^{1 / 3}}, \quad \mu_{1}=\mu_{0}\left(\frac{g^{2}}{v}\right)^{1 / 3}, \quad \alpha^{2}=\frac{(v g)^{1 / 3}}{\rho g} .
\end{gathered}
$$

And here $u=u(y, t), S_{x y}=S_{x y}(y, t), S_{x x}=S_{x x}(y, t)$ are the dimensionless velocity and the dimensionless deviatoric stress tensor; $\alpha^{2}, S_{1}, S_{2}$, and $\mu_{1}$ represent the porous medium constant, dimensionless relaxation and retardation time constants, and a dimensionless material 
parameter, respectively. No slip at the wall $y=0$ and zero shear rate on the free surface of the liquid are assumed,

$$
u(0, t)=0, \quad t \geq 0, \quad \frac{\partial u}{\partial y}=0 \quad \text { on } y=h .
$$

The liquid is at rest at $t=0$, therefore initial conditions are

$$
u(y, 0)=S_{x y}(y, 0)=S_{x x}(y, 0)=0 .
$$

To calculate the shape of the film profile at a given time $t$, the thickness $h$ is allowed to vary with $x$ while assuming the flow is still locally parallel. Combining the material derivative at the free surface

$$
v(x, h, t)=\frac{\partial h}{\partial t}+u(x, h, t) \frac{\partial h}{\partial x}
$$

with the equation of continuity yields a differential equation in $h$,

$$
-\frac{\partial h}{\partial x}\left(\int_{0}^{h} \frac{\partial u}{\partial h} d y+u\right)=\frac{\partial h}{\partial t} .
$$

Introducing the flow rate $Q(h, t)$ per unit width across the film thickness $h$,

$$
Q(h, t)=\int_{0}^{h} u(x, y, t) d y,
$$

differentiating $Q$ with respect to $h$, and substituting the result into (2.8) and integrating give

$$
x(h, t)-x_{0}(h)=\int_{0}^{t} \frac{\partial Q}{\partial h} d \tau
$$

where $x_{0}(h)=x(h, 0)$ is the initial profile and can be chosen to represent any suitable initial shape. Equation (2.10) effectively determines the position of the free surface $x$ as a function of $h$ and $t$.

Next some mathematical notation is introduced. Denote the inner product in $\mathbb{L}^{2}(0, h)$ by

$$
(f, g)=\int_{0}^{h} f(y) g(y) d y
$$

If $f \in \mathbb{L}^{2}(0, H)$, then Fourier sine series is defined as

$$
f(y)=\sum_{k=1}^{\infty} \widehat{f}_{s}(k) \sin \left(\left(\frac{(2 k-1) \pi}{2 h}\right) y\right),
$$


where

$$
\widehat{f}_{s}(k)=\frac{2}{h} \int_{0}^{\pi} \sin \left(\frac{(2 k-1) \pi y}{2 h}\right) f(y) d y \quad k=1,2, \ldots
$$

Similarly, Fourier cosine series is defined as

$$
f(y)=\frac{\widehat{f}_{c}(0)}{2}+\sum_{k=1}^{\infty} \widehat{f}_{c}(k) \cos \left(\left(\frac{(2 k-1) \pi}{2 h}\right) y\right),
$$

where

$$
\widehat{f}_{c}(k)=\frac{2}{H} \int_{0}^{H} \cos \left(\frac{(2 k-1) \pi y}{2}\right) f(y) d y \quad k=1,2, \ldots
$$

Denote by \|\|$_{H^{m}}$ the Sobolev norm, given by

$$
\|f\|_{H^{m}}^{2}=\left\{\begin{array}{l}
\sum_{k=1}^{\infty}\left(1+\left|\frac{2 k-1}{2}\right|^{2}\right)^{m}\left|\widehat{f}_{s}(k)\right|^{2}, \\
\frac{\left|\widehat{f}_{c}(0)\right|^{2}}{2}+\sum_{k=1}^{\infty}\left(1+\left|\frac{2 k-1}{2}\right|^{2}\right)^{m}\left|\widehat{f}_{c}(k)\right|^{2} .
\end{array}\right.
$$

The space of periodic Sobolev functions on the interval $[0, h]$ is defined as the closure of the space of smooth periodic functions with respect to the $H^{m}$-norm and will be simply denoted by $H^{m}$. In particular, the space $\mathbb{L}^{2}(0, h)$ with norm denoted by \|\|$_{\mathbb{L}^{2}}$ is recovered for $m=0$. We now define subspaces of $\mathbb{L}^{2}(0, h)$ spanned by the set

$$
\begin{aligned}
& D_{N}^{*}=\left\{\frac{\sqrt{2} \sin ((2 k-1) \pi y / 2 h)}{\sqrt{h}}, 1 \leq k \leq N\right\}, \\
& D_{N}^{* *}=\left\{\frac{\sqrt{2} \cos ((2 k-1) \pi y / 2 h)}{\sqrt{h}}, 1 \leq k \leq N\right\} .
\end{aligned}
$$

The operators $P_{N}$ and $P_{N}^{*}$ denote the orthogonal, self-adjoint projection of $\mathbb{L}^{2}$ onto $D_{N}^{*}$ and $D_{N}^{* *}$ defined, respectively, by

$$
\begin{aligned}
& P_{N} f(y)=\sum_{k=1}^{N} \sin \left(\frac{(2 k-1) \pi y}{2 h}\right) \widehat{f}_{s}(k), \\
& P_{N}^{*} f(y)=\frac{\widehat{f}_{c}(0)}{2}+\sum_{k=1}^{N} \cos \left(\frac{(2 k-1) \pi y}{2 h}\right) \widehat{f}_{c}(k) .
\end{aligned}
$$


For $f \in H^{m}$, the estimates:

$$
\begin{gathered}
\left\|f-P_{N} f\right\|_{L^{2}} \leq C_{p} N^{-m}\left\|\partial_{y}^{m} f\right\|_{L^{2^{\prime}}} \\
\left\|f-P_{N} f\right\|_{H^{n}} \leq C_{p} N^{n-m}\left\|\partial_{y}^{m} f\right\|_{L^{2^{\prime}}}
\end{gathered}
$$

hold for an appropriate constant $C_{p}$ and a positive integer $n$. The reader is referred to [8] for the proof of these inequalities.

The space of continuous functions from the interval $[0, T]$ into the space $H^{n}$ is denoted by $C\left([0, T], H^{n}\right)$. Similarly, we also consider the space $C\left([0, T], D_{N}^{*}\right)$, where the topology on the finite-dimensional space $D_{N}^{*}$ can be given by any norm. Finally, note the inverse inequality

$$
\left\|\partial_{y}^{m} \varphi\right\|_{L^{2}} \leq N^{m}\|\varphi\|_{L^{2}}
$$

which holds for integers $m>0$ and $\varphi \in D_{N}^{*}$. A proof of this estimate can also be found in [11]. We will make use of the Sobolev lemma, which guarantees the existence of a constant $c$ such that

$$
\sup _{y}|f(y)| \leq c\|f\|_{H^{1}}
$$

We now note that exactly the same estimates hold for $P_{N}^{*}$. In the following, it will always be assumed that a solution of our problem (2.11)-(2.15) exists on some time interval $[0, T]$ with a certain amount of spatial regularity. In particular, we suppose that a solution exists in the $\left(C\left([0, T], H^{1}\right)\right)^{3}$ space for some $T>0$. With these preliminaries in place, we are now set to tackle the problem of defining a suitable spectral projection of $(2.11)-(2.15)$ and proving the convergence of such a projection. First, the Fourier-Galerkin method is presented and a proof of convergence given.

\section{The Fourier-Galerkin Method}

$\left\{e_{k}(y), k \in \mathbb{N}\right\}=\{\sqrt{2} \sin ((2 k-1) \pi y / 2 h) / \sqrt{h}, k \in \mathbb{N}\}$ and $\left\{f_{k}(y), k \in \mathbb{N}\right\}=\{\sqrt{2} \cos ((2 k-$ 1) $\pi y / 2 h) / \sqrt{h}, k \in \mathbb{N}\}$ are chosen to be an orthonormal basis of the Hilbert space $\mathbb{L}_{0}^{2}[0, h]$ and $\mathbb{L}^{2}[0, h]$, respectively. Then, the subspace of these Hilbert spaces spanned by the $D_{N}^{*}=$ $\{\sqrt{2} \sin ((2 k-1) \pi y / 2 h) / \sqrt{h}, 1 \leq k \leq N\}$ and $D_{N}^{* *}=\{\sqrt{2} \cos ((2 k-1) \pi y / 2 h) / \sqrt{h}, 0 \leq$ $k \leq N\}$, respectively. Fourier-Galerkin approximation of (2.1)-(2.5) are find the functions $u^{N}(t) \in D_{N^{\prime}}^{*} S_{x y}^{N}(t)$, and $S_{x x}^{N} \in D_{N}^{* *}$ for all $0 \leq t \leq T$, such that

$$
\begin{gathered}
\left(\partial_{t} u^{N}-1-\partial_{Y} S_{x y}^{N}, \omega_{1}+\beta^{2} u, \omega_{1}\right)=0, \quad t \in[0, T] \\
\left(S_{x y}^{N}+S_{1} \partial_{t} S_{x y}^{N}+\frac{1}{2} \mu_{1} S_{x x}^{N} \partial_{y} u^{N}-\partial_{y} u^{N}-S_{2} \partial_{t y}^{2} u^{N}, \omega_{2}\right)=0, \quad t \in[0, T],
\end{gathered}
$$




$$
\begin{gathered}
\left(S_{x x}^{N}+S_{1} \partial_{t} S_{x x}^{N}-2 S_{1} S_{x y}^{N} \partial_{y} u^{N}-2 S_{2}\left(\partial_{y} u^{N}\right)^{2}, \omega_{2}\right)=0, \quad t \in[0, T] \\
u^{N}(0)=0, \quad S_{x y}^{N}(0)=0, \quad S_{x x}^{N}(0)=0,
\end{gathered}
$$

for all $\omega_{1} \in D_{N}^{*}$ and for all $\omega_{2} \in D_{N}^{* *}$. Since for each $t, u^{N}(\cdot, t), S_{x y}^{N}(\cdot, t)$, and $S_{x y}^{N}(\cdot, t)$ have the form

$$
\begin{aligned}
& u^{N}(y, t)=\sum_{k=1}^{N} \widehat{u}^{N}(k, t) \frac{\sqrt{2} \sin ((2 k-1) \pi y / 2 h)}{\sqrt{h}}, \\
& S_{x y}^{N}(y, t)=\sum_{k=1}^{N} \widehat{S}_{x y}^{N}(k, t) \frac{\sqrt{2} \cos ((2 k-1) \pi y / 2 h)}{\sqrt{h}}, \\
& S_{x x}^{N}(y, t)=\sum_{k=1}^{N} \widehat{s}_{x x}^{N}(k, t) \frac{\sqrt{2} \cos ((2 k-1) \pi y / 2 h)}{\sqrt{h}} .
\end{aligned}
$$

Taking $\omega_{1}=\sqrt{2 / H} \sin ((2 k-1) \pi y / 2 h), \omega_{2}=\sqrt{2 / H} \cos ((2 k-1) \pi y / 2 h), 1 \leq k \leq$ $N$ in (3.1)-(3.3) yields the following system of equations for the Fourier coefficients of $u^{N}$, $S_{x y}^{N}$, and $S_{x x}^{N}$ :

$$
\begin{aligned}
\frac{d}{d t} \widehat{u}^{N}(k, t)= & -\frac{(2 k-1) \pi}{2 h} \widehat{s}_{x y}^{N}(k, t)+\sqrt{\frac{2}{h} \frac{2 h}{\pi(2 k-1)}} \\
\widehat{s}_{x y}^{N}(k, t)+S_{1} \frac{d}{d t} \widehat{s}_{x y}^{N}(k, t)= & \frac{(2 k-1) \pi}{2 h} \widehat{u}^{N}(k, t)+S_{2} \frac{(2 k-1) \pi}{2 h} \frac{d}{d t} \widehat{u}^{N}(k, t) \\
& +\mu_{1} \frac{(2 k-1) \pi}{4 h} \sqrt{\frac{2}{h}} \sum_{i, j=1}^{N} c_{i j N} \widehat{s}_{x x}^{N}(i, t) \widehat{u}^{N}(k, t), \\
\widehat{s}_{x x}^{N}(k, t)+S_{1} \frac{d}{d t} \widehat{s}_{x x}^{N}(k, t)-\frac{(2 k-1) \pi}{2 h} \widehat{u}^{N}(k, t) & -2 S_{1} \frac{(2 k-1) \pi}{4 h} \sqrt{\frac{2}{h}} \sum_{i, j=1}^{N} c_{i j N} \widehat{s}_{x y}^{N}(i, t) \widehat{u}^{N}(k, t) \\
= & -2 S_{2}\left(\frac{(2 k-1) \pi}{4 h}\right){ }^{2} \sqrt{\frac{2}{h}} \sum_{i, j=1}^{N} d_{i j N} \widehat{u}^{N}(i, t) \widehat{u}^{N}(j, t), \\
\widehat{u}^{N}(k, t)=0, & \widehat{s}_{x y}^{N}(k, t)=0, \\
c_{i j N}= & \int_{0}^{h} \cos \left(\frac{(2 i-1) \pi y}{2 h}\right) \sin \left(\frac{(2 j-1) \pi y}{2 h}\right) \cos \left(\frac{(2 N-1) \pi y}{2 h}\right) d y, \\
d_{i j N}= & \int_{0}^{h} \sin \left(\frac{(2 i-1) \pi y}{2 h}\right) \sin \left(\frac{(2 j-1) \pi y}{2 h}\right) \cos \left(\frac{(2 N-1) \pi y}{2 h}\right) d y .
\end{aligned}
$$


This is a nonlinear system of ordinary differential equations for the functions $\left\{\widehat{u}^{N}(k, t), \widehat{s}_{x y}^{N}(k, t), \widehat{s}_{x x}^{N}(k, t)\right\}_{k=1}^{N} ;$ by standard existence theory, there is a unique solution which exists on some time interval $\left[0, T_{N}\right)$, where $T_{N}$ possibly may be equal to $T$. Since the argument is standard, the proof is omitted here. The main result of this paper is the fact that the Galerkin approximation $\left\{u^{N}, S_{x y}^{N}, S_{x x}^{N}\right\}$ converges to the exact solution $\left\{u, S_{x y}, S_{x x}\right\}$ when $u$ is smooth enough. This is stated in the next theorem.

Theorem 3.1. Suppose that a solution $\left\{u, S_{x y}, S_{x x}\right\}$ of (2.1)-(2.5) exists in the space $\left(C\left([0, T], H^{m}\right)\right)^{3}$ for $m \geq 1$ and for some time $T>0$. If $\left\|P_{N} u(h, t)-u^{N}(u, t)\right\| \leq c N^{1-m}$ and $\left\|P_{N}^{*} S_{x y}-S_{x y}^{N}\right\| \leq c N^{1-m}$, then, for large enough $N$, there exists a unique solution $\left\{u_{N}, S_{x y}^{N}, S_{x x}^{N}\right\}$ of the finite dimensional problem (3.1)-(3.4). Moreover, there exist constants $\Gamma_{1}, \Gamma_{2}$, and $\Gamma_{3}$ such that

$$
\begin{gathered}
\sup _{t \in[0, T]}\left\|u-u^{N}\right\|_{L^{2}} \leq \Gamma_{1} N^{1-m}, \quad \sup _{t \in[0, T]}\left\|S_{x y}-S_{x y}^{N}\right\|_{L^{2}} \leq \Gamma_{2} N^{1-m}, \\
\sup _{t \in[0, T]}\left\|S_{x x}-S_{x x}^{N}\right\|_{L^{2}} \leq \Gamma_{3} N^{1-m} .
\end{gathered}
$$

Before the proof is given, note that the assumptions of the theorem encompass the existence of constants $\kappa, \kappa_{1}$, and $\kappa_{2}$ such that

$$
\sup _{t \in[0, T]}\|u(y, t)\|_{H^{m}} \leq \kappa, \quad \sup _{t \in[0, T]}\left\|S_{x x}(y, t)\right\|_{H^{m}} \leq \kappa_{1}, \quad \sup _{t \in[0, T]}\left\|S_{x y}(y, t)\right\|_{H^{m}} \leq \kappa_{2} .
$$

In particular, it follows that there are other constants $\psi, \psi_{1}$, and $\psi_{2}$ such that

$$
\sup _{t \in[0, T]}\|u(y, t)\|_{H^{m}} \leq \psi, \quad \sup _{t \in[0, T]}\left\|S_{x x}(y, t)\right\|_{H^{m}} \leq \psi_{1}, \quad \sup _{t \in[0, T]}\left\|S_{x y}(y, t)\right\|_{H^{m}} \leq \psi_{2} .
$$

The main ingredient in the proof of the theorem is a local error estimate which will be established by the following lemma.

Lemma 3.2. Suppose that the solution $\left\{u^{N}, S_{x y}^{N}, S_{x x}^{N}\right\}$ of (3.1)-(3.4) exists on the time interval $\left[0, t_{N}^{*}\right]$ and that $\left.\sup _{t \in\left[0, t_{N}^{*}\right]}\left\|u^{N}(y, t)\right\|_{H^{2}} \leq 2 \psi, \sup _{t \in\left[0, t_{N}^{*}\right.}\right]\left\|S_{x y}^{N}(y, t)\right\|_{H^{2}} \leq 2 \psi_{1}$, $\sup _{t \in\left[0, t_{N}^{*}\right]}$ $\left\|S_{x x}^{N}(y, t)\right\|_{H^{2}} \leq 2 \psi_{2}, \sup _{t \in\left[0, t_{N}^{*}\right]}\left|P_{N} u(h, t)-u^{N}(h, t)\right| \leq \beta N^{1-m}$, and $\sup _{t \in\left[0, t_{N}^{*}\right]} \mid P_{N}^{*} S_{x y}(h, t)-$ $S_{x y}^{N}(h, t) \mid \leq \beta_{1} N^{1-m}$, then the error estimate:

$$
\begin{gathered}
\sup _{t \in\left[0, t_{N}^{*}\right]}\left\|u-u^{N}\right\|_{L^{2}} \leq \Gamma_{1} N^{1-m}, \quad \sup _{t \in\left[0, t_{N}^{*}\right]}\left\|S_{x y}-S_{x y}^{N}\right\|_{L^{2}} \leq \Gamma_{2} N^{1-m}, \\
\sup _{t \in\left[0, t_{N}^{*}\right]}\left\|S_{x x}-S_{x x}^{N}\right\|_{L^{2}} \leq \Gamma_{3} N^{1-m}
\end{gathered}
$$

holds for constant $\Gamma_{1}$ which is the function of $T, \alpha^{2}, C_{p}, \kappa, \beta$, and $c$, constant $\Gamma_{2}$ which is the function of $T, S_{1}, S_{2}, \kappa_{1}, \psi, \psi_{1}, \psi_{2}, c_{1}, \beta_{1}$, and $c$, and constant $\Gamma_{3}$ which is the function of $T, S_{1}, \kappa_{1}, \psi, \psi_{1}, c_{1}, \kappa$, and $c$. 
Proof. Let $\sigma_{1}=P_{N} u-u^{N}, \sigma_{2}=P_{N}^{*} S_{x y}-S_{x y}^{N}, \sigma_{3}=P_{N}^{*} S_{x x}-S_{x x}^{N}$. Also, from the definition of $P_{N}$ and $P_{N}^{*}$, we have $\partial_{y} \sigma_{1}=P_{N}^{*} \partial_{y} u-\partial_{y} u^{N}, \partial_{y} \sigma_{2}=P_{N} \partial_{y} S_{x y}-\partial_{y} S_{x y^{\prime}}^{N} \partial_{y} \sigma_{3}=P_{N} \partial_{y} S_{x x}-\partial_{y} S_{x x}^{N}$. We apply $P_{N}, P_{N}^{*}$ and $P_{N}^{*}$ to both sides of (2.1)-(2.3), respectively. Since $P_{N}, P_{N}^{*}$ commute with derivation, we obtain

$$
\begin{gathered}
\partial_{t} P_{N} u+\alpha^{2} u=1+\partial_{y} P_{N} S_{x y} \\
P_{N}^{*} S_{x y}+S_{1} \partial_{t} P_{N}^{*} S_{x y}+\frac{1}{2} \mu_{1} P_{N}^{*}\left(S_{x x} \partial_{y} u\right)=P_{N}^{*} \partial_{y} u+S_{2} \partial_{t} P_{N}^{*} \partial_{y} u, \\
P_{N}^{*} S_{x x}+S_{1} \partial_{t} P_{N}^{*} S_{x x}-2 S_{1} P_{N}^{*}\left(S_{x y} \partial_{y} u\right)=-2 S_{2} P_{N}^{*}\left(\partial_{y} u\right)^{2} .
\end{gathered}
$$

We multiply these equations with test functions $\sigma_{1} \in D_{N^{\prime}}^{*} \sigma_{2} \in D_{N^{\prime}}^{* *}$ and $\sigma_{3} \in D_{N^{\prime}}^{* *}$ respectively, integrate over $[0, h]$, and subtract the resulting expressions from (3.1), (3.2), and (3.3) to get

$$
\begin{aligned}
& \frac{1}{2} \frac{d}{d t}\left\|\sigma_{1}\right\|_{L^{2}}^{2}+\alpha^{2}\left\|\sigma_{1}\right\|_{L^{2}}^{2}=\left(P_{N} 1-1_{N}, \sigma_{1}\right)+\left(P_{N} \partial_{y}\left(S_{x y}-S_{x y}^{N}\right), \sigma_{1}\right) \\
& \left\|\sigma_{2}\right\|_{L^{2}}^{2}+S_{1} \frac{1}{2} \frac{d}{d t}\left\|\sigma_{2}\right\|_{L^{2}}^{2}+\frac{1}{2} \mu_{1}\left(P_{N}^{*}\left(S_{x x} \partial_{y} u\right)-S_{x x}^{N} \partial_{y} u^{N}, \sigma_{2}\right) \\
& =\left(P_{N}^{*} \partial_{y}\left(u-u^{N}\right), \sigma_{2}\right)+S_{2}\left(P_{N}^{*} \partial_{t y}^{2}\left(u-u^{N}\right), \sigma_{2}\right) \\
& \left\|\sigma_{2}\right\|_{L^{2}}^{2}+S_{1} \frac{1}{2} \frac{d}{d t}\left\|\sigma_{2}\right\|_{L^{2}}^{2}+\frac{1}{2} \mu_{1}\left(P_{N}^{*}\left(S_{x x} \partial_{y} u\right)-S_{x x}^{N} \partial_{y} u^{N}, \sigma_{2}\right) \\
& =\left(P_{N}^{*} \partial_{y}\left(u-u^{N}\right), \sigma_{2}\right)+S_{2}\left(\partial_{t} P_{N}^{*} \partial_{y}\left(u-u^{N}\right), \sigma_{2}\right) \\
& \left\|\sigma_{3}\right\|_{L^{2}}^{2}+S_{1} \frac{1}{2} \frac{d}{d t}\left\|\sigma_{3}\right\|_{L^{2}}^{2}-2 S_{1}\left(P_{N}^{*}\left(S_{x y} \partial_{y} u\right)-S_{x y}^{N} \partial_{y} u^{N}, \sigma_{3}\right) \\
& =-2 S_{2}\left(P_{N}^{*}\left(\partial_{y} u\right)^{2}-\left(\partial_{y} u^{N}\right)^{2}, \sigma_{3}\right) .
\end{aligned}
$$

Since $\sigma_{1} \in D_{N^{\prime}}^{*}\left(\sigma_{2}, \sigma_{3}\right) \in D_{N^{\prime}}^{* *}$

$$
\frac{1}{2} \frac{d}{d t}\left\|\sigma_{1}\right\|_{L^{2}}^{2}+\alpha^{2}\left\|\sigma_{1}\right\|_{L^{2}}^{2}=\left(\partial_{y} \sigma_{2}, \sigma_{1}\right) \leq\left\|\partial_{y} \sigma_{2}\right\|_{L^{2}}\left\|\sigma_{1}\right\|_{L^{2}}
$$

then we have

$$
\frac{1}{2} \frac{d}{d t}\left\|\sigma_{1}\right\|_{L^{2}}^{2}+\alpha^{2}\left\|\sigma_{1}\right\|_{L^{2}}^{2} \leq\left\|\partial_{y} \sigma_{2}\right\|_{L^{2}} \leq\left|\sigma_{2}(H, t)\right|
$$


Consequently, from hypothesis and Gronwall's inequality, we obtain

$$
\sup _{t \in\left[0, t_{N}^{*}\right]}\left\|\sigma_{1}\right\|_{L^{2}} \leq \Gamma_{1} N^{1-m}
$$

where $\Gamma_{1}$ is a function of $T, \beta, \alpha^{2}$, and $c$ :

$$
\left\|\sigma_{2}\right\|_{L^{2}}^{2}+S_{1} \frac{1}{2} \frac{d}{d t}\left\|\sigma_{2}\right\|_{L^{2}}^{2}+\frac{1}{2} \mu_{1}\left(S_{x x} \partial_{y} u-S_{x x}^{N} \partial_{y} u^{N}, \sigma_{2}\right)=\left(\partial_{y} \sigma_{1}, \sigma_{2}\right)+S_{2}\left(\partial_{t y}^{2} \sigma_{1}, \sigma_{2}\right)
$$

Hence, we get

$$
\begin{gathered}
\left\|\sigma_{2}\right\|_{L^{2}}^{2}+S_{1} \frac{1}{2} \frac{d}{d t}\left\|\sigma_{2}\right\|_{L^{2}}^{2}+\frac{1}{2} \mu_{1}\left(S_{x x} \partial_{y} u-S_{x x}^{N} \partial_{y} u^{N}, \sigma_{2}\right) \\
\leq\left\|\partial_{y} \sigma_{1}\right\|_{L^{2}}\left\|\sigma_{2}\right\|_{L^{2}}+S_{2}\left\|\partial_{t y}^{2} \sigma_{1}\right\|_{L^{2}}\left\|\sigma_{2}\right\|_{L^{2}} \\
\left\|\sigma_{3}\right\|_{L^{2}}^{2}+S_{1} \frac{1}{2} \frac{d}{d t}\left\|\sigma_{3}\right\|_{L^{2}}^{2}-2 S_{1}\left(S_{x y} \partial_{y} u-S_{x y}^{N} \partial_{y} u^{N}, \sigma_{3}\right) \\
=-2 S_{2}\left(\left(\partial_{y} u\right)^{2}-\left(\partial_{y} u^{N}\right)^{2}, \sigma_{3}\right) .
\end{gathered}
$$

Let us estimate third term on the left-hand side of (3.20) in the time interval $\left[0, T_{N}\right)$ :

$$
\begin{aligned}
\left(S_{x x} \partial_{y} u-S_{x x}^{N} \partial_{y} u^{N}, \sigma_{2}\right)= & \left(\left(S_{x x}-S_{x x}^{N}\right)\left(\partial_{y}\left(u+u^{N}\right)\right)\right. \\
& \left.+S_{x x}^{N}\left(\partial_{y}\left(u-u^{N}\right)\right)+\partial_{y} u^{N}\left(\left(S_{x x}-S_{x x}^{N}\right), \sigma_{2}\right)\right) .
\end{aligned}
$$

Thus,

$$
\begin{aligned}
& \left|\left(S_{x x} \partial_{y} u-S_{x x}^{N} \partial_{y} u^{N}, \sigma_{2}\right)\right| \leq \sup _{y}\left|\partial_{y}\left(u+u^{N}\right)\right|\left\|S_{x x}-S_{x x}^{N}\right\|_{L^{2}}\left\|\sigma_{2}\right\|_{L^{2}} \\
& \quad+\sup _{y}\left|S_{x x}^{N}\right|\left\|\partial_{y}\left(u-u^{N}\right)\right\|_{L^{2}}\left\|\sigma_{2}\right\|_{L^{2}}+\sup _{y}\left|\partial_{y} u^{N}\right|\left\|S_{x x}-S_{x x}^{N}\right\|_{L^{2}}\left\|\sigma_{2}\right\|_{L^{2}} \\
& \leq\left(c\left\|u+u^{N}\right\|_{H^{2}}+c\left\|u^{N}\right\|_{H^{2}}\right)\left(\left\|S_{x x}-P_{N} S_{x x}\right\|_{L^{2}}+\left\|P_{N} S_{x x}-S_{x x}^{N}\right\|_{L^{2}}\right)\left\|\sigma_{2}\right\|_{L^{2}} \\
& \quad+c\left\|S_{x x}^{N}\right\|_{H^{1}}\left(\left\|u-P_{N} u\right\|_{H^{1}}+\left\|P_{N} u-u^{N}\right\|_{H^{1}}\right)\left\|\sigma_{2}\right\|_{L^{2}} \leq 5 c \psi \\
& \quad \times\left(C_{p} N^{-m}\left\|S_{x x}\right\|_{H^{m}}+\left\|\sigma_{2}\right\|_{L^{2}}\right)\left\|\sigma_{2}\right\|_{L^{2}}+2 c \bigwedge_{1}\left(C_{p} N^{1-m}\|u\|_{H^{m}}+\left\|\sigma_{2}\right\|_{L^{2}}\right)\left\|\sigma_{2}\right\|_{L^{2}} .
\end{aligned}
$$


Hence,

$$
\begin{aligned}
& \left\|\sigma_{2}\right\|_{L^{2}}^{2}+S_{1} \frac{d}{d t}\left\|\sigma_{2}\right\|_{L^{2}} \leq \psi_{2}\left\|\sigma_{2}\right\|_{L^{2}}+5 c \psi \kappa N^{-m}+2 c \kappa_{1} \psi_{1} C_{p} N^{1-m}+\left\|\partial_{y} \sigma_{1}\right\|_{L^{2}} \\
& \leq\left(\psi_{2}-1\right)\left\|\sigma_{2}\right\|_{L^{2}}+5 c \psi C_{p} \kappa N^{-m}+2 c \kappa_{1} \psi_{1} C_{p} N^{1-m}+\left\|\partial_{y} \sigma_{1}\right\|_{L^{2}} \\
& \leq c_{1}\left\|\sigma_{2}\right\|_{L^{2}}+c N^{1-m}+\left\|\partial_{y} \sigma_{1}\right\|_{L^{2}}+S_{2}\left\|\partial_{t y}^{2} \sigma_{1}\right\|_{L^{2}} .
\end{aligned}
$$

Using the hypothesis and Gronwall's inequality,

$$
\sup _{t \in\left[0, t_{N}^{*}\right]}\left\|\sigma_{2}\right\|_{L^{2}} \leq \Gamma_{2} N^{1-m}
$$

$\Gamma_{1}$ is a function of $T, S_{1}, S_{2}, \kappa_{1}, \psi, \psi_{1}, \psi_{2}, c_{1}, \beta_{1}$, and $c$. Estimating the RHS of (3.22) in the time interval $\left[0, T_{N}\right)$,

$$
\begin{aligned}
\left(\left(\partial_{y} u\right)^{2}-\left(\partial_{y} u^{N}\right)^{2}, \sigma_{3}\right)= & \left(\partial_{y}\left\{\left(u+u^{N}\right)\left(u-u^{N}\right)\right\}, \sigma_{3}\right) \\
= & \left(\partial_{y}\left(u+u^{N}\right)\left(u-u^{N}\right), \sigma_{3}\right)+\left(\left(u+u^{N}\right) \partial_{y}\left(u-u^{N}\right), \sigma_{3}\right) \\
= & \left(\partial_{y}\left(u+u^{N}\right)\left(u-u^{N}\right), \sigma_{3}\right)+\left(\left(u+u^{N}\right) \partial_{y}\left(u-P_{N} u\right), \sigma_{3}\right) \\
& +\left(\left(u+u^{N}\right) \partial_{y}\left(P_{N} u-u^{N}\right), \sigma_{3}\right), \\
\left|\left(\partial_{y} u\right)^{2}-\left(\partial_{y} u^{N}\right)^{2}, \sigma_{3}\right| \leq & \sup _{y}\left|\partial_{y}\left(u+u^{N}\right)\right|\left\|u-u^{N}\right\|_{L^{2}}\left\|\sigma_{3}\right\|_{L^{2}} \\
& +\sup _{y}\left|\partial_{y}\left(u+u^{N}\right)\right|\left\|\partial_{y}\left(u-P_{N} u\right)\right\|_{L^{2}}\left\|\sigma_{3}\right\|_{L^{2}} \\
& +\left|\int_{0}^{H}\left(u+u^{N}\right) \partial_{y}\left(\sigma_{3}\right) \sigma_{3} d y\right| \\
\leq & c\left\|u+u^{N}\right\|_{H^{2}}\left(\left\|u-P_{N} u\right\|_{L^{2}}+\left\|P_{N} u-u^{N}\right\|_{L^{2}}\right)\left\|\sigma_{3}\right\|_{L^{2}} \\
& +c\left\|u+u^{N}\right\|_{H^{1}}\left\|u-P_{N} u\right\|_{H^{1}}\left\|\sigma_{3}\right\|_{L^{2}}+\frac{1}{2} \int_{0}^{H} \sigma_{3}^{2}\left|\partial_{y}\left(u+u^{N}\right) d y\right| \\
\leq & 3 c \psi\left(C_{p} N^{-m}\|u\|_{H^{m}}+\left\|\sigma_{3}\right\|_{L^{2}}\right)\left\|\sigma_{3}\right\|_{L^{2}} \\
& +3 c \psi C_{p} N^{1-m}\|u\|_{H^{m}}\left\|\sigma_{3}\right\|_{L^{2}}+\frac{1}{2} \sup _{y}\left|\partial_{y}\left(u+u^{N}\right)\right| \int_{0}^{H} \sigma_{3}^{2} d y .
\end{aligned}
$$

Noting that the last integral is bounded by $(1 / 2) 3 c \psi$, the estimate is

$$
\left|\left(\partial_{y} u\right)^{2}-\left(\partial_{y} u^{N}\right)^{2}, \sigma_{3}\right| \leq 3 c \psi\left\|\sigma_{3}\right\|_{L^{2}}\left(\frac{3}{2}\left\|\sigma_{3}\right\|_{L^{2}}+C_{p}\|u\|_{H^{m}}\left(N^{-m}+N^{1-m}\right)\right) .
$$


$\left(S_{x y} \partial_{y} u-S_{x y}^{N} \partial_{y} u^{N}, \sigma_{3}\right)$ can be estimated in exactly the same way as $\left(S_{x x} \partial_{y} u-S_{x x}^{N} \partial_{y} u^{N}, \sigma_{2}\right)$. Then, (3.22) as a whole is estimated as

$$
\begin{aligned}
\left\|\sigma_{3}\right\|_{L^{2}}+S_{1} \frac{d}{d t}\left\|\sigma_{3}\right\|_{L^{2}} \leq & \left(\psi_{2}\left\|\sigma_{3}\right\|_{L^{2}}+5 c \psi C_{p} \kappa N^{-m}+2 c \kappa_{1} \psi_{1} C_{p} N^{1-m}\right) \\
& +3 c \psi\left(\frac{3}{2}\left\|\sigma_{3}\right\|_{L^{2}}+C_{p} \kappa\left(N^{-m}+N^{1-m}\right)\right) .
\end{aligned}
$$

Therefore, we get

$$
c_{1}\left\|\sigma_{3}\right\|_{L^{2}}+S_{1} \frac{d}{d t}\left\|\sigma_{3}\right\|_{L^{2}} \leq c N^{1-m}
$$

Then, using the Gronwall's inequality,

$$
\sup _{t \in\left[0, t_{N}^{*}\right]}\left\|\sigma_{3}\right\|_{L^{2}} \leq \Gamma_{3} N^{1-m},
$$

where $\Gamma_{3}$ is a function of $T, S_{1}, \kappa_{1}, \psi, \psi_{1}, c_{1}$, and $c$. Since

$$
\begin{aligned}
\left\|u-u^{N}\right\|_{L^{2}} & =\left\|u+P_{N} u-P_{N} u-u^{N}\right\|_{L^{2}} \\
& \leq\left\|u-P_{N} u\right\|_{L^{2}}+\left\|P_{N} u-u^{N}\right\|_{L^{2}} \\
\left\|S_{x y}-S_{x y}^{N}\right\|_{L^{2}} & =\left\|S_{x y}+P_{N}^{*} S_{x y}-P_{N}^{*} S_{x y}-S_{x y}^{N}\right\|_{L^{2}} \\
& \leq\left\|S_{x y}-P_{N}^{*} S_{x y}\right\|_{L^{2}}+\left\|P_{N}^{*} S_{x y}-S_{x y}^{N}\right\|_{L^{2}} \\
\left\|S_{x x}-S_{x x}^{N}\right\|_{L^{2}} & =\left\|S_{x x}+P_{N}^{*} S_{x x}-P_{N}^{*} S_{x x}-S_{x x}^{N}\right\|_{L^{2}} \\
& \leq\left\|S_{x x}-P_{N}^{*} S_{x x}\right\|_{L^{2}}+\left\|P_{N}^{*} S_{x x}-S_{x x}^{N}\right\|_{L^{2}} .
\end{aligned}
$$

Using (2.18) and (3.19) in (3.33), (2.18) and (3.26) in (3.34), (2.18) and (3.32) in (3.35), respectively, we obtain

$$
\begin{gathered}
\sup _{t \in\left[0, t_{N}^{*}\right]}\left\|u-u^{N}\right\|_{L^{2}} \leq \Gamma_{1} N^{1-m}, \sup _{t \in\left[0, t_{N}^{*}\right]}\left\|S_{x y}-S_{x y}^{N}\right\|_{L^{2}} \leq \Gamma_{2} N^{1-m}, \\
\sup _{t \in\left[0, t_{N}^{*}\right]}\left\|S_{x x}-S_{x x}^{N}\right\|_{L^{2}} \leq \Gamma_{3} N^{1-m},
\end{gathered}
$$

where $\Gamma_{1}, \Gamma_{2}$, and $\Gamma_{3}$ are constants, functions of $\left(T, \alpha^{2}, C_{p}, \kappa, \beta\right.$, and $\left.c\right),\left(T, S_{1}, S_{2}, \kappa_{1}\right.$, $\psi, \psi_{1}, \psi_{2}, c_{1}, \beta_{1}$, and $\left.c\right)$, and of $\left(T, S_{1}, \kappa_{1}, \psi, \psi_{1}, c_{1}, \kappa\right.$, and $\left.c\right)$, respectively. 


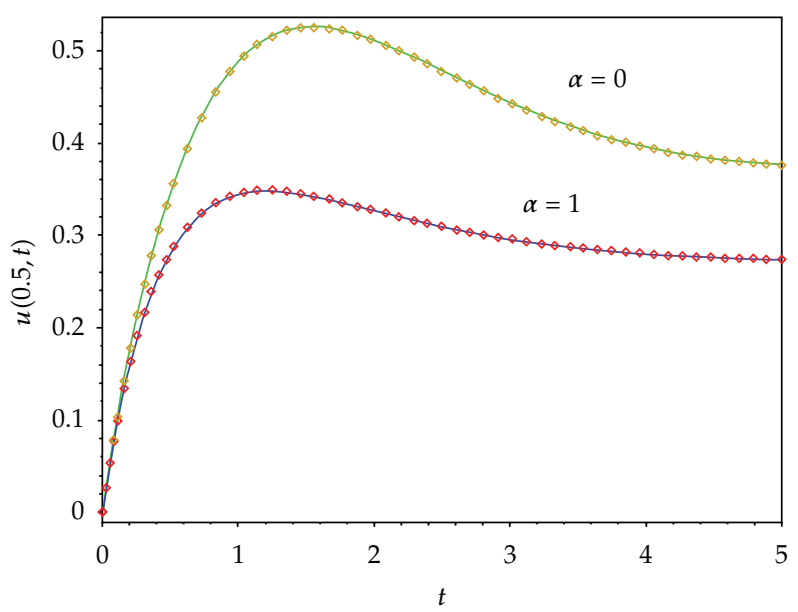

Figure 1: Comparison of exact analytical and numerical solutions of centerline velocity field for $\mu_{1}=0$, $S_{1}=2, S_{2}=1$, and $h=1$ for both permeable wall $(\alpha=1)$ and impermeable wall.

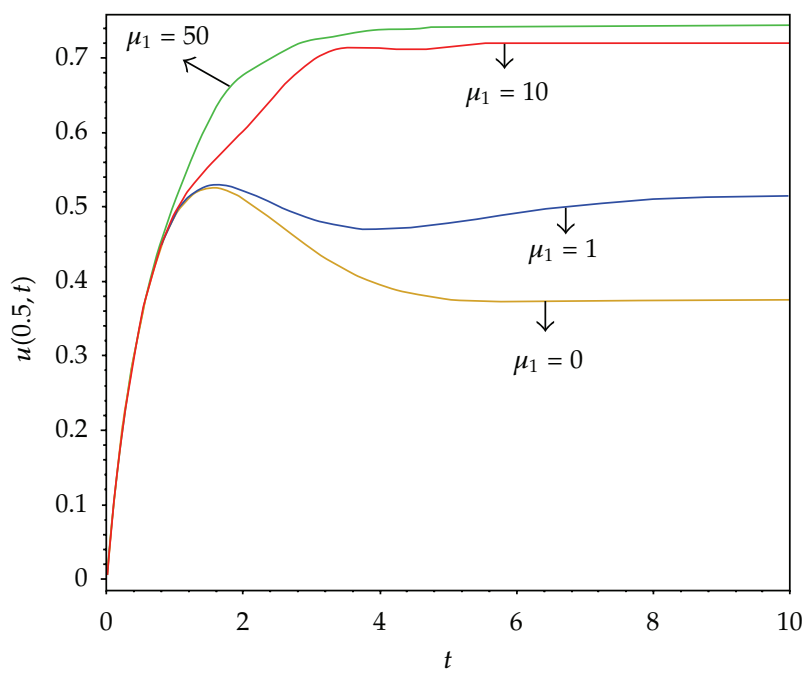

Figure 2: The effect of the nonlinear parameter $\mu_{1}$ on the centerline velocity profile for $S_{1}=2, S_{2}=1, \alpha=0$, and $h=1$.

Lemma 3.3. Suppose that the solution $\left\{u^{N}, S_{x y}^{N}, S_{x x}^{N}\right\}$ of (3.1)-(3.3) exists on the time interval $\left[0, t_{N}^{*}\right]$ and that $\sup _{t \in\left[0, t_{N}^{*}\right]}\left\|u^{N}(y, t)\right\|_{H^{2}} \leq 2 \psi, \sup _{t \in\left[0, t_{N}^{*}\right]}\left\|S_{x y}^{N}(y, t)\right\|_{H^{2}} \leq 2 \psi_{1}, \sup _{t \in\left[0, t_{N}^{*}\right]}$ $\left\|S_{x x}^{N}(y, t)\right\|_{H^{2}} \leq 2 \psi_{2}, \sup _{t \in\left[0, t_{N}^{*}\right]}\left|P_{N} u(h, t)-u^{N}(h, t)\right| \leq \beta N^{1-m}$, and $\sup _{t \in\left[0, t_{N}^{*}\right]} \mid P_{N}^{*} S_{x y}(h, t)-$ $S_{x y}^{N}(h, t) \mid \leq \beta_{1} N^{1-m}$, then the error estimate:

$$
\begin{gathered}
\sup _{t \in\left[0, t_{N}^{*}\right]}\left\|u-u^{N}\right\|_{H^{2}} \leq \Gamma_{1} N^{3-m}, \sup _{t \in\left[0, t_{N}^{*}\right]}\left\|S_{x y}-S_{x y}^{N}\right\|_{H^{2}} \leq \Gamma_{2} N^{3-m}, \\
\sup _{t \in\left[0, t_{N}^{*}\right]}\left\|S_{x x}-S_{x x}^{N}\right\|_{H^{2}} \leq \Gamma_{3} N^{3-m},
\end{gathered}
$$




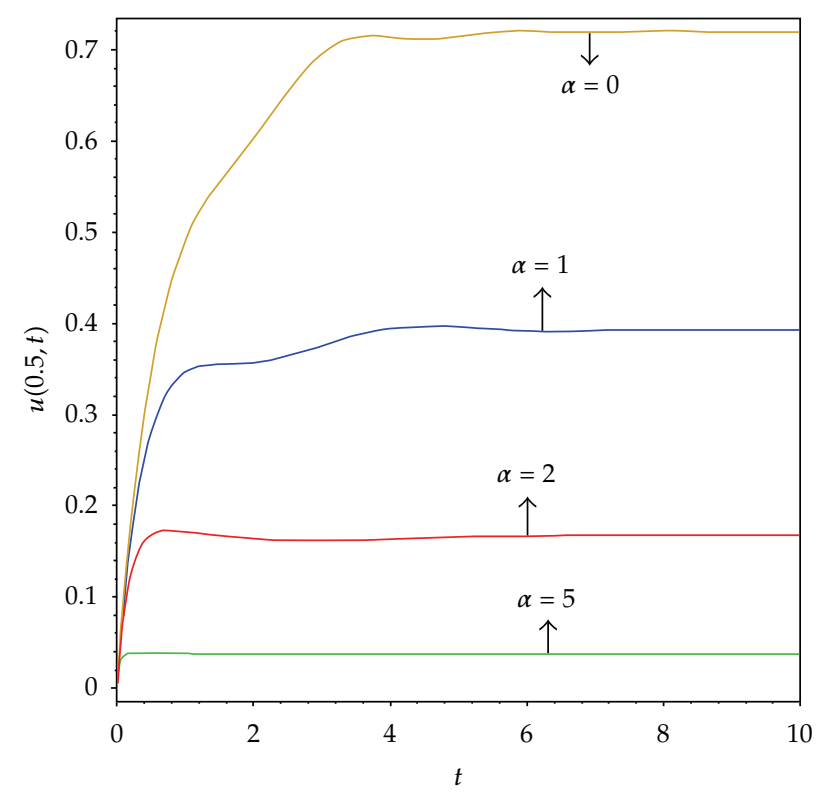

Figure 3: The effect of the porous medium parameter $\alpha^{2}$ on the centerline velocity profile for $S_{1}=2, S_{2}=1$, $\mu_{1}=10$, and $h=1$.

holds for the constants $\Gamma_{1}, \Gamma_{2}$, and $\Gamma_{3}$. The proof of the Lemma follows from (3.19), (3.26), and (3.32) after application of the triangle inequality and the inverse inequality (2.19).

Proof of Theorem 3.1. To extend the estimate of the first inequality in (3.14) to the time interval $[0, T] t_{N}^{*}$ unspecified in Lemma 3.2 is defined as

$$
t_{N}^{*}=\sup \left\{t \in[0, T] \mid \text { for all } t^{\prime} \leq t,\left\|u^{N}\left(y, t^{\prime}\right)\right\|_{H^{2}} \leq 2 \psi\right\} .
$$

Thus, the time $t_{N}^{*}$ corresponds to the largest time in $[0, T]$ for which the $H^{2}$-norm of $u_{N}$ is uniformly bounded by $2 \psi$. Since $\left\|u^{N}(y, 0)\right\|_{H^{2}}=\left\|P_{N}(y, 0)\right\|_{H^{2}}$,

$$
\left\|u^{N}(y, 0)\right\|_{H^{2}} \leq\|u(y, 0)\|_{H^{2}} \leq \psi,
$$

therefore $t_{N}^{*}>0$ for all $N$. Note that $t_{N}^{*}$ is smaller than the maximum time of existence of the solution $T_{N}$. Now, we need to show that there exists $N^{L}$ such that

$$
t_{N}^{*}=T \quad \forall N \geq N^{L},
$$

and therefore the supremum in (3.14) holds on $[0, T]$. From the definition (3.38), we either have $t_{N}^{*}=T$ or $t_{N}^{*}<T$ in which case $\left\|u^{N}\left(y, t^{\prime}\right)\right\|_{H^{2}}=2 \psi$. Now assume that $t_{N}^{*}<T$, then

$$
\begin{aligned}
2 \psi & =\left\|u^{N}\left(y, t_{N}^{*}\right)\right\|_{H^{2}} \leq\left\|u^{N}\left(y, t_{N}^{*}\right)-u(y, t)\right\|_{H^{2}}+\|u(y, t)\|_{H^{2}} \\
& =\left\|u^{N}\left(y, t_{N}^{*}\right)-u(y, t)\right\|_{H^{2}}+\psi .
\end{aligned}
$$




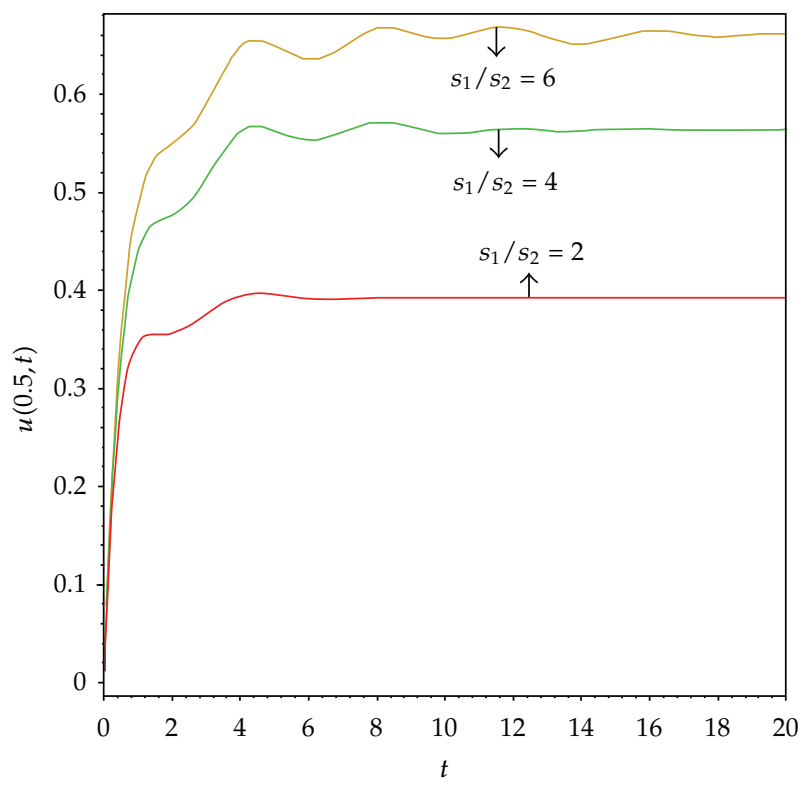

Figure 4: The effect of the elasticity on the centerline velocity profile for $\mu_{1}=10$ and $h=1$ for permeable wall $(\alpha=1)$.

Hence, we obtain

$$
\psi \leq\left\|u^{N}\left(y, t_{N}^{*}\right)-u(y, t)\right\|_{H^{2}}
$$

On the other hand, Lemma 3.3 implies

$$
\psi \leq \Gamma_{1} N^{3-m}
$$

or

$$
N \leq\left(\frac{\Gamma_{1}}{\psi}\right)^{1 / m-3}
$$

In conclusion, for $N^{L}>\left(\Gamma_{1} / \psi\right)^{1 / m-3}$, we cannot have $t_{N}^{*}<T$ and claim (3.40) holds. It follows that $N \geq N^{L}$ the solution $u_{N}$ of (3.1) is defined on $[0, T]$, since as noted before $t_{N}^{*}<T_{N}$, and, from (3.14),

$$
\sup _{t \in[0, T]}\left\|u(y, t)-u^{N}(y, t)\right\|_{L^{2}} \leq \Gamma_{1} N^{1-m}
$$




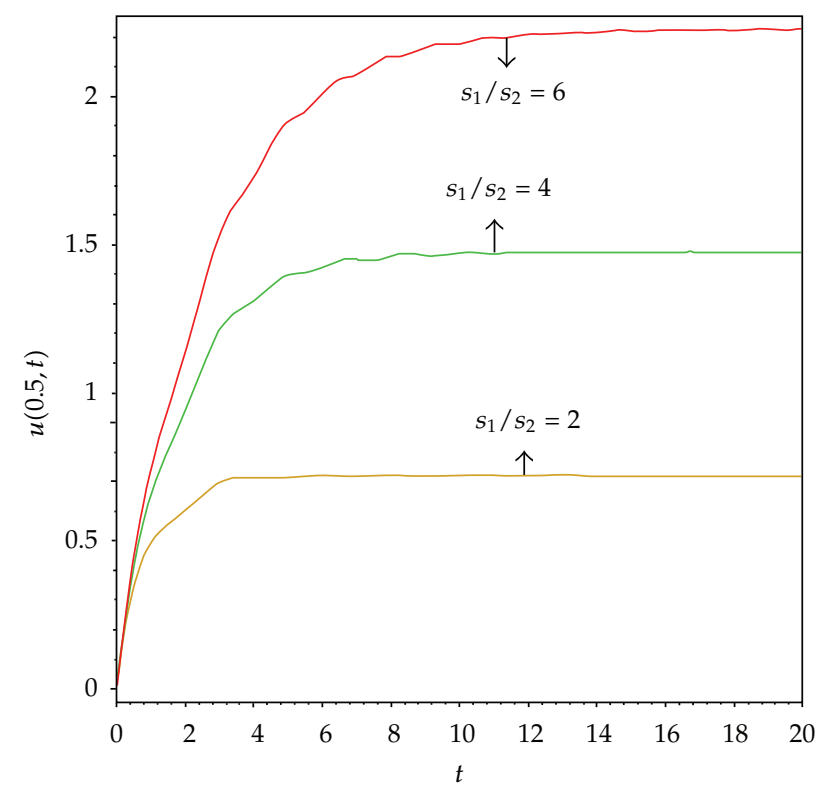

Figure 5: The effect of the elasticity on the centerline velocity profile for $\mu_{1}=10$ and $h=1$ for impermeable wall.

In exactly the same way, we can extend the estimate of the second and third inequalities in (3.14) to the time interval $[0, T]$ and show that

$$
\begin{aligned}
& \sup _{t \in[0, T]}\left\|S_{x y}(y, t)-S_{x y}^{N}(y, t)\right\|_{L^{2}} \leq \Gamma_{2} N^{1-m}, \\
& \sup _{t \in[0, T]}\left\|S_{x x}(y, t)-S_{x x}^{N}(y, t)\right\|_{L^{2}} \leq \Gamma_{3} N^{1-m} .
\end{aligned}
$$

\section{Numerical Results and Discussion}

The system of differential equations (3.6)-(3.9) is of the following form

$$
\begin{gathered}
\frac{d}{d t} \widehat{u}^{N}(k, t)=G_{1}\left(\widehat{s}_{x y}^{N}(k, t)\right), \\
\frac{d}{d t} \widehat{s}_{x y}^{N}(k, t)=G_{2}\left(\widehat{s}_{x x}^{N}(i, t), \hat{s}_{x y}^{N}(k, t), \widehat{u}^{N}(k, t), S_{1}, S_{2}\right), \\
\frac{d}{d t} \widehat{s}_{x x}^{N}(k, t)=G_{3}\left(\widehat{s}_{x x}^{N}(i, t), \widehat{s}_{x y}^{N}(k, t), \widehat{u}^{N}(k, t), S_{1}, S_{2}\right), \\
\widehat{u}^{N}(k, t)=0, \quad \widehat{s}_{x y}^{N}(k, t)=0, \quad \widehat{s}_{x x}^{N}(k, t)=0 .
\end{gathered}
$$

Runge-Kutta method is applied to this system. The integrals in equations (2.8) and (2.9) are calculated analytically and numerically, respectively, with $\partial Q / \partial h$ approximated by a central difference formula. To illustrate the spectral accuracy, the time step is chosen to be sufficiently 


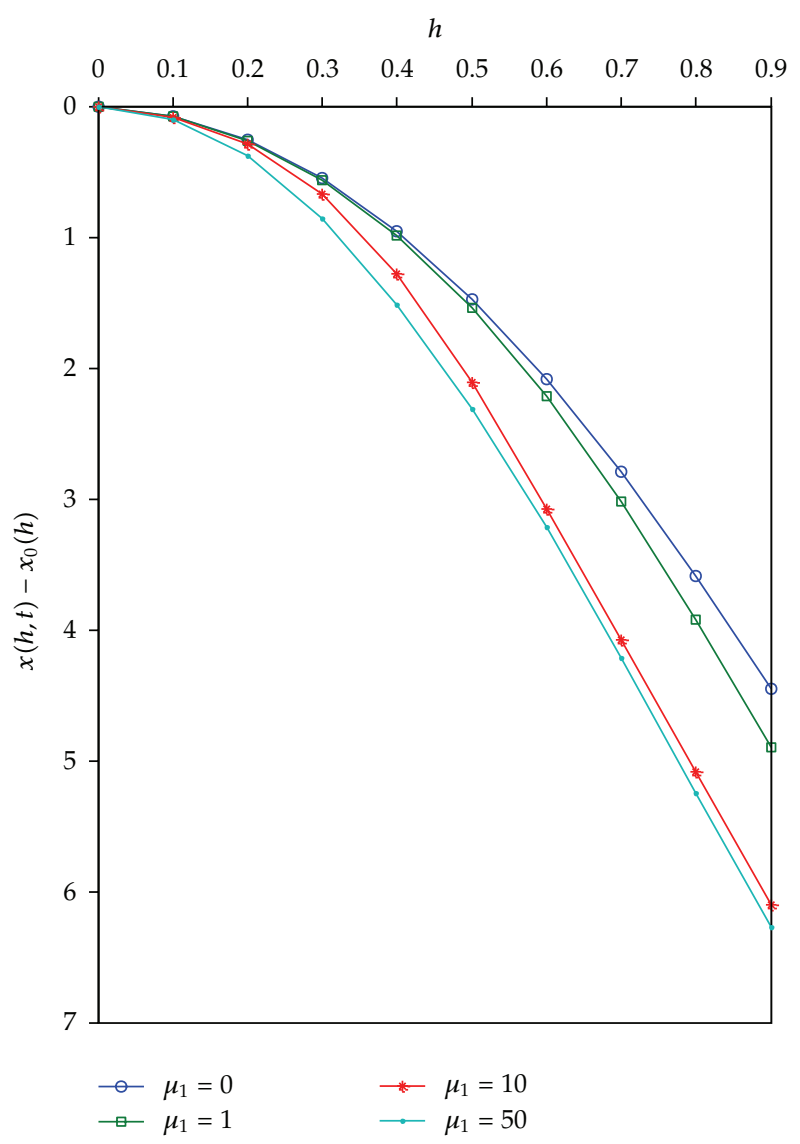

Figure 6: The effect of the material parameter $\mu_{1}$ on the drainage rate for $S_{1}=2, S_{2}=1$ for impermeable wall.

Table 1

\begin{tabular}{lcc}
\hline$N$ (number of modes) & $\log _{10}$ (error) impermeable wall & $\log _{10}$ (error) permeable wall $(\alpha=1)$ \\
\hline 10 & -5.1 & -5.3 \\
15 & -6.45 & -6.55 \\
20 & -7.12 & -7.14 \\
\hline
\end{tabular}

small so that the error is dominated by the spatial discretization. The free drainage of the Oldroyd-B liquid $\left(\mu_{1}=0\right)$ for which an exact analytical solution is possible is considered first [14]. Figure 1 compares the exact analytical solution in [14] with the approximate solution with $N=5$ nodes only for both permeable and impermeable wall. The exact and approximate solutions are indistinguishable in the figure. The error $\log _{10}\left(\left\|u^{N}-u\right\|_{L^{\infty}[0, h]}\right)$ at $t=1$ of the Fourier-Galerkin approximations with increasing number of nodes for the drainage of Oldroyd-B liquid is listed in Table 1.

This shows that numerical results are at least accurate up to the seventh decimal for $N=20$. The aim of this paper is to elaborate the effects of the nonlinear parameter $\mu_{1}$ and porous medium parameter $\alpha^{2}$ on the centerline velocity and drainage rate. The effect of these 


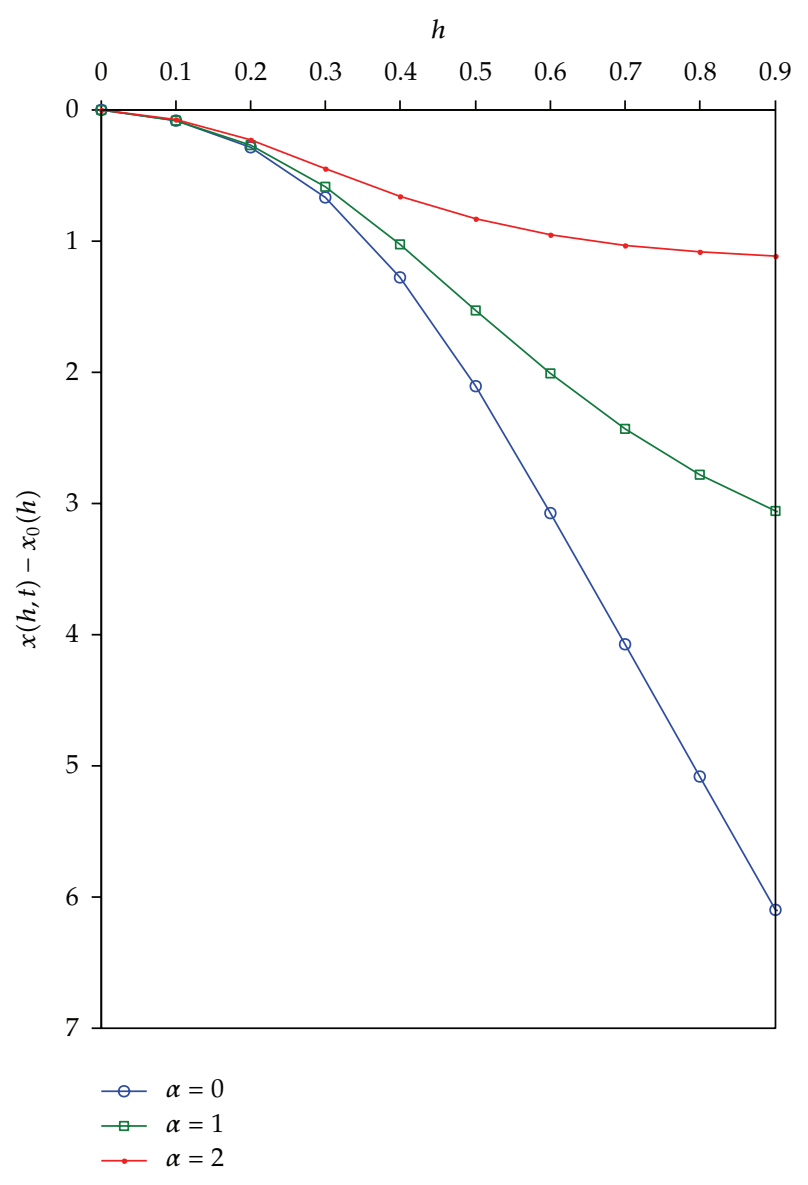

Figure 7: The effect of porous medium parameter $\alpha^{2}$ on the drainage rate for $S_{1}=2, S_{2}=1$, and $\mu_{1}=10$.

parameters on the velocity field is shown in Figures 2 and 3. Figure 2 displays the effect of the nonlinear parameter $\mu_{1}$ on the centerline velocity when the wall is impermeable $\alpha=0$, and $S_{1}=2, S_{2}=1$, and $h=1$. Clearly, the overshoot gradually disappears as numerical values of the nonlinear parameter $\mu_{1}$ increase. In addition, the steady centerline velocity increases with increasing values of $\mu_{1}$. Figure 3 explores the effect of porosity on the centerline velocity for $\mu_{1}=10$. Increasing the porosity parameter triggers a decrease in the value of the centerline velocity. The difference between the relaxation and retardation times, $S_{1}-S_{2}$, is a measure of the elasticity of the Oldroyd four-constant liquid, the greater the difference is the more elastic the liquid is. The effect of elasticity on the centerline velocity of constant viscosity Oldroyd four-constant liquids is shown in Figures 4 and 5 for permeable and impermeable walls, respectively. In either case, the centerline velocity increases with an increase in elasticity. The effect of the nonlinear parameter $\mu_{1}$ and porous medium parameter $\alpha^{2}$ on the drainage rate is examined in Figures 6 and 7, respectively. Since in all cases the nonlinear parameter $\mu_{1}$ has an increasing effect on the velocity, we expect increasing $\mu_{1}$ will lead to a thinner film over either type of wall, impermeable or permeable. That is evident in Figure 6, which shows that liquid drain more rapidly as $\mu_{1}$ is increased from zero. Since the porous medium parameter $\alpha^{2}$ has a decreasing effect on the velocity in all cases (both permeable and impermeable wall), we expect increasing $\alpha^{2}$ will lead to a thicker film over either impermeable or permeable wall, 


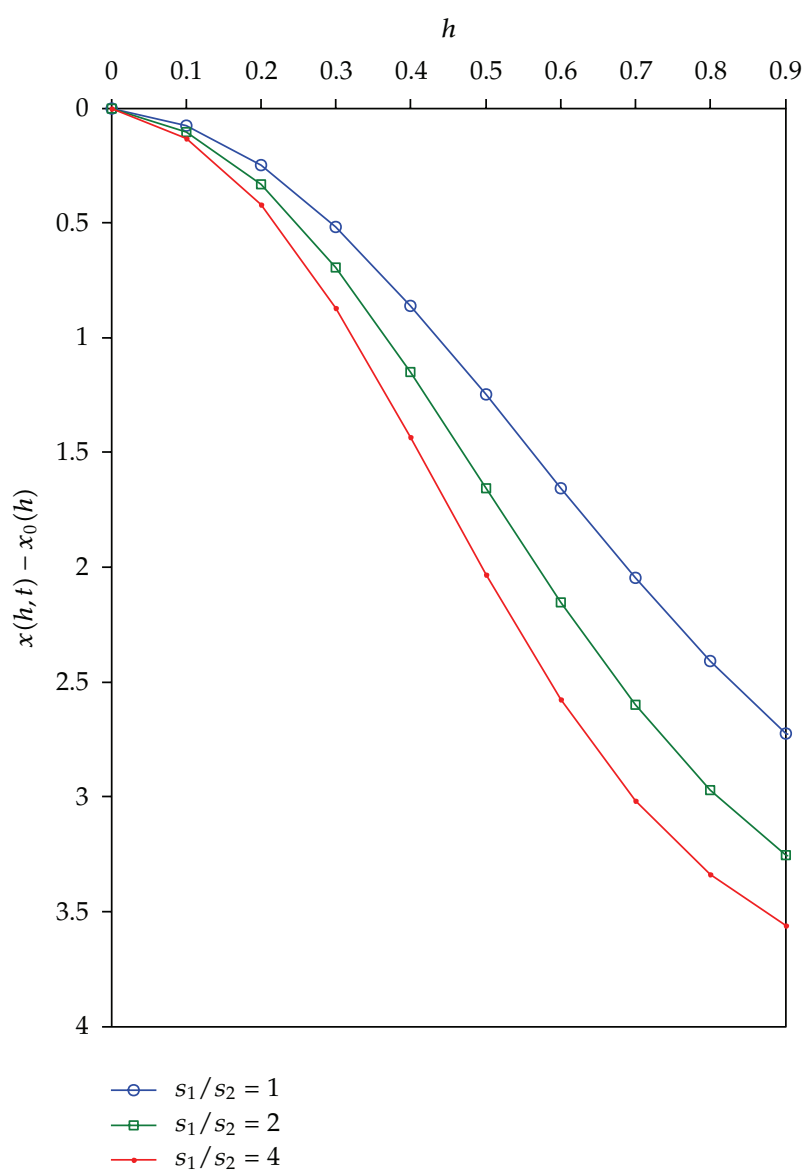

Figure 8: The effect of elasticity on the drainage rate for $\alpha=1$ and $\mu_{1}=1$.

Figure 7. The effect of the elasticity on the drainage rate is shown in Figure 8 for three liquids of which liquid 1 is the most elastic and liquid 3 is the least elastic. Liquid 1 drains more rapidly than liquid 2, which in turn drains more rapidly than liquid 3.

\section{References}

[1] J. Q. Xu, "Modeling unsteady-state gravity-driven flow in porous media," Journal of Petroleum Science and Engineering, vol. 62, no. 3-4, pp. 80-86, 2008.

[2] J. A. Goshawk, N. D. Waters, G. K. Rennie, and E. J. Staples, "Enhancement of the drainage of nonNewtonian liquid films by oscillation," Journal of Non-Newtonian Fluid Mechanics, vol. 51, no. 1, pp. 21-60, 1994.

[3] J. A. Goshawk and N. D. Waters, "The effect of oscillation on the drainage of an elastico-viscous liquid," Journal of Non-Newtonian Fluid Mechanics, vol. 54, pp. 449-464, 1994.

[4] S. V. Pennington, N. D. Waters, and E. W. Williams, "The numerical simulation of an oldroyd liquid draining down a vertical surface," Journal of Non-Newtonian Fluid Mechanics, vol. 34, no. 2, pp. 221-239, 1990.

[5] A. M. Keeley, G. K. Rennie, and N. D. Waters, "Draining thin films-part 1," Journal of Non-Newtonian Fluid Mechanics, vol. 28, no. 2, pp. 213-226, 1988.

[6] Y. Dimakopoulos and J. Tsamopoulos, "On the gas-penetration in straight tubes completely filled with a viscoelastic fluid," Journal of Non-Newtonian Fluid Mechanics, vol. 117, no. 2-3, pp. 117-139, 2004. 
[7] M. Pavlidis, Y. Dimakopoulos, and J. Tsamopoulos, "Fully developed flow of a viscoelastic film down a vertical cylindrical or planar wall," Rheologica Acta, vol. 48, no. 9, pp. 1031-1048, 2009.

[8] Y. Maday and A. Quarteroni, "Error analysis for spectral approximation of the Korteweg-de Vries equation," RAIRO Modélisation Mathématique et Analyse Numérique, vol. 22, no. 3, pp. 499-529, 1988.

[9] H. Kalisch and X. Raynaud, "Convergence of a spectral projection of the Camassa-Holm equation," Numerical Methods for Partial Differential Equations, vol. 22, no. 5, pp. 1197-1215, 2006.

[10] A. Quarteroni and A. Valli, Numerical Approximation of Partial Differential Equations, Springer, Berlin, Germany, 1997.

[11] C. Canuto, M. Y. Hussaini, A. Quarteroni, and T. A. Zang, Spectral Methods in Fluid Dynamics, Springer, New York, NY, USA, 1988.

[12] F. T. Akyildiz, K. Vajravelu, and H. Ozekes, "Fourier-Galerkin domain truncation method for Stokes' first problem with Oldroyd four-constant liquid," Computers and Mathematics with Applications, vol. 55, no. 11, pp. 2452-2457, 2008.

[13] N. D. Waters and A. M. Keeley, "Start-up of an elastico-viscous liquid draining from a vertical surface," Journal of Non-Newtonian Fluid Mechanics, vol. 22, no. 3, pp. 325-334, 1987.

[14] F. T. Akyildiz and D. A. Siginer, "Start-up of an elastico-viscous liquid draining from a porous vertical surface," Journal of Non-Newtonian Fluid Mechanics. In press. 


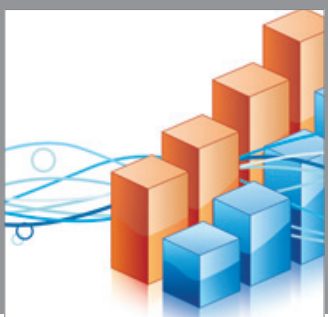

Advances in

Operations Research

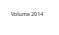

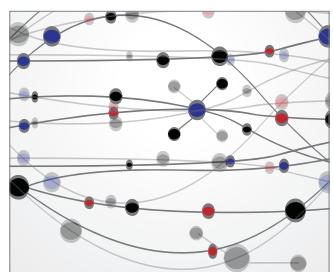

\section{The Scientific} World Journal
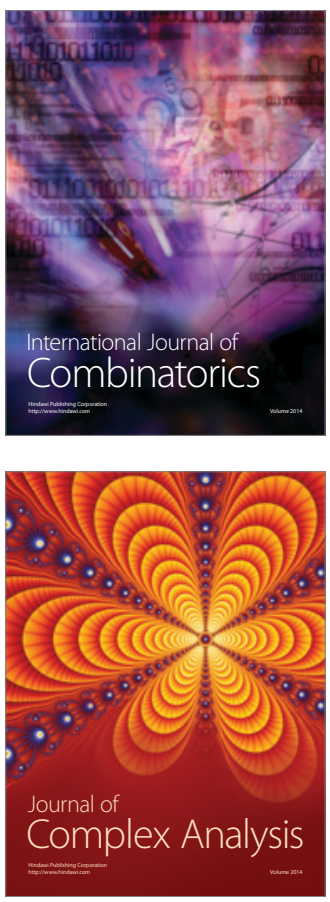

International Journal of

Mathematics and

Mathematical

Sciences
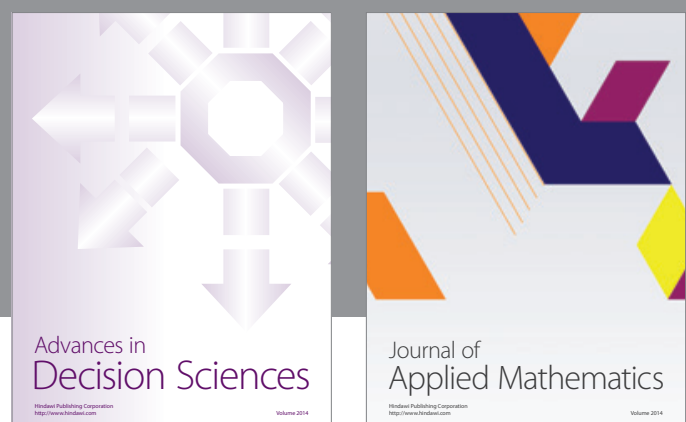

Journal of

Applied Mathematics
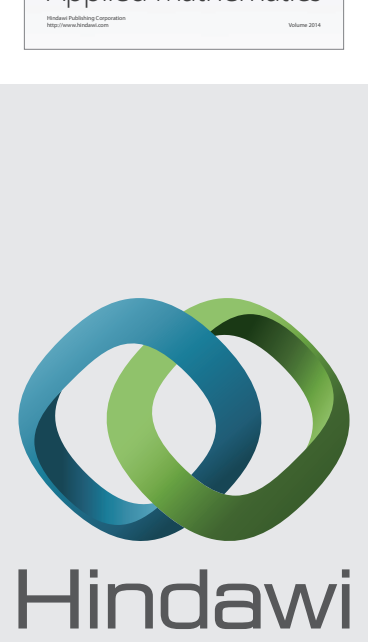

Submit your manuscripts at http://www.hindawi.com
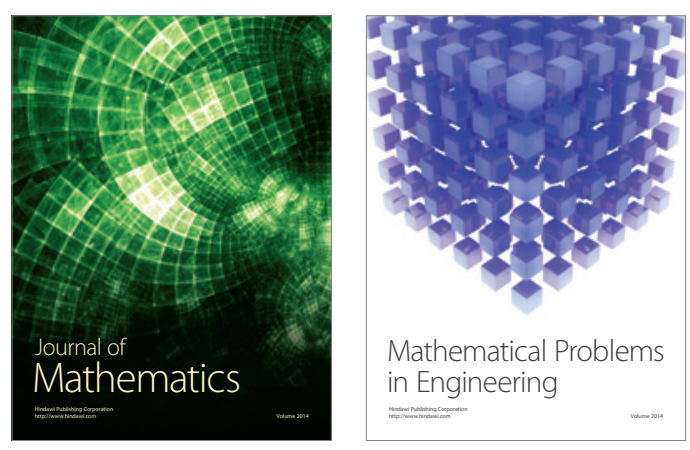

Mathematical Problems in Engineering
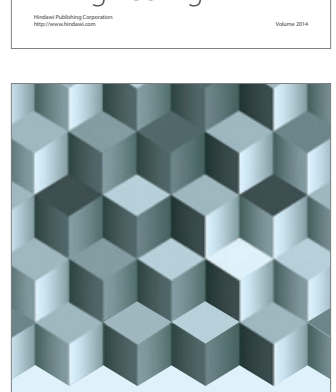

Journal of

Function Spaces
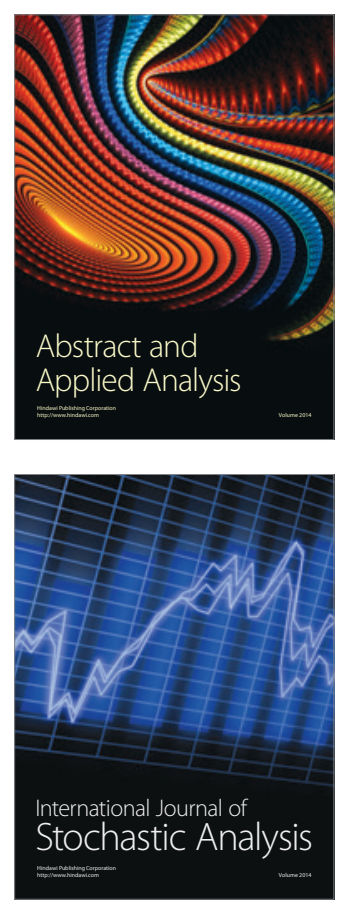

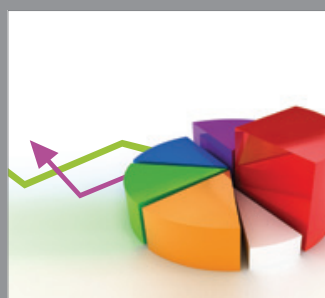

ournal of

Probability and Statistics

Promensencen
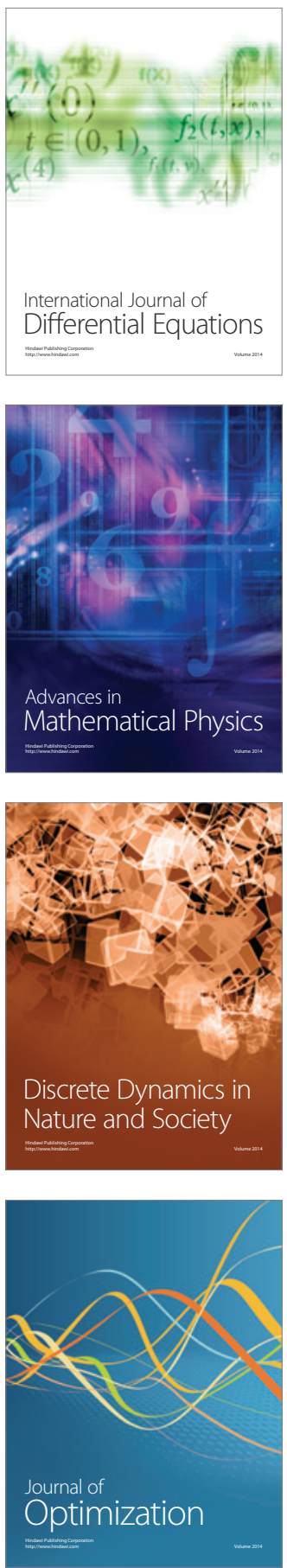